\title{
Epigenetic Silencing of $O R$ and TAS2R Genes Expression in Human Orbitofrontal Cortex At Early Stages of Sporadic Alzheimer's Disease
}

Victoria Cunha Alves ( $\nabla$ vdacunhaalves@gmail.com )

Research Institute Hospital 12 de Octubre

Joana Figueiro-Silva

Research Institute Hospital 12 de Octubre

Isidre Ferrer

Institut d'Investigació Biomédica de Bellvitge

Eva Carro

Research Institute Hospital 12 de Octubre

\section{Research Article}

Keywords: Modulation, neurological, disease, epigenetic

Posted Date: December 3rd, 2021

DOI: https://doi.org/10.21203/rs.3.rs-1098461/v1

License: (a) (i) This work is licensed under a Creative Commons Attribution 4.0 International License.

Read Full License 


\section{Abstract}

Modulation of brain olfactory $(O R)$ and taste receptors (TASR) expression was recently reported in neurological diseases. We explored the possible expression and regulation of selected $O R$ and TASR genes in human orbitofrontal cortex of sporadic Alzheimer's disease (AD) and found that these are expressed and markedly downregulated at early stages. The expression pattern did not follow disease progression suggesting regulation through epigenetic mechanisms. We found an increase of global H3K9me3 levels and substantial enrichment of this repressive signature at ORs and TAS2Rs proximal promoter at early stages, ultimately lost at advanced stages. By mass spectrometry-based proteomic and further validation, we found that $\mathrm{H} 3 \mathrm{~K} 9 \mathrm{me} 3$ interacts with MeCP2 at early stages and that this protein is increased in sporadic AD. Findings suggest MeCP2 might be implicated in $O R$ and TAS2R genes expression regulation through interaction with $\mathrm{H} 3 \mathrm{~K} 9 \mathrm{me}$, and as an early event, it may uncover a novel etiopathogenetic mechanism of sporadic AD.

\section{Introduction}

Alzheimer's disease (AD) is the most prevalent late-life dementia, yet it currently lacks an effective treatment. The accrual of pathological alterations featured in multiple brain regions includes accumulation of neurotoxic amyloid $\beta$ peptide $(A \beta)$, tau protein hyperphosphorylation, synaptic failure, diffuse neuronal loss, inflammation, and mitochondrial dysfunction ${ }^{1}$. The staging patterns of tau neurofibrillary tangles (NFT) pathology remains a consistent indicator of disease progression, manifesting in six distinct stages generalized into transentorhinal stages (stage I-II), limbic stages (stage $\mathrm{III}-\mathrm{IV}$ ), and isocortical stages (stage $\mathrm{V}-\mathrm{VI}$ ) ${ }^{2,3}$.

Age-related cognitive decline involves the prefrontal cortex, a vulnerable brain region implicated in memory, emotion, cognitive functions, decision making, and social behavior ${ }^{4}$. Although poorly understood, the orbitofrontal cortex (OFC) is regarded as one of the most polymodal regions of the brain and an important site of highly multisensory convergence ${ }^{5}$. Remarkably, the OFC is directly interconnected with the amygdala, acting as part of the complex limbic system. In advanced stages of $A D$, disruption of the temporo-amygdala-orbitofrontal network manifests with cognitive and behavioral symptoms, which are not present if the pathology is limited to the hippocampocentric division ${ }^{6}$.

Olfactory receptors (ORs) are seven-transmembrane G protein-coupled chemoreceptors (GPCRs) expressed primarily in olfactory epithelium. However, in recent years, reports surfaced of its widespread expression in a variety of non-chemosensory tissues ${ }^{7}$. In human brain, ORs expression was found in cerebral cortex, thalamus, in certain nuclei of the brainstem and in cerebellar Purkinje cells ${ }^{8-11}$.

Taste receptors (TASRs) are chemoreceptors that reside primarily in specialized taste receptor cells within taste buds. Some type 2 TASRs (TAS2R3, TAS2R5, TAS2R13) are narrowly tuned to structurally similar bitter compounds, whereas others are broadly tuned (TAS2R14, TAS2R39, TAS2R46), responding to several bitter compounds ${ }^{12}$. There is only limited evidence of TASRs expression in human brain. TAS2Rs 
(TAS2R4, TAS2R5, TAS2R10, TAS2R13, TAS2R14, TAS2R39, TAS2R50) expression was detected in human cerebral cortex ${ }^{8,10,11}$ and in choroid plexus ${ }^{13}$, whereas type 1 TASRs (TAS1R) expression was found in rat choroid plexus ${ }^{14}$ and in murine hypothalamus, hippocampus, and cortex, wherein these receptors were suggested to operate as membrane-bound brain glucose sensors ${ }^{15}$.

Thus far, the transcriptional regulation of $O R$ and TASR genes expression in humans still represents a fundamental open question.

The human genome contains 3 protein-coding TAS1R and 25 protein-coding TAS2R genes, and almost 400 different protein-coding $O R$ genes. The $O R$ gene superfamily comprises 18 families and 300 subfamilies, and putatively functional $O R$ genes are located in multiple clusters scattered throughout nearly all chromosomes. TAS1R genes are located within a single cluster on chromosome 1 , whereas functional TAS2R genes are mostly localized in clusters on chromosome $7 q 31$ and 12 p13 ${ }^{16}$.

In this study, we sought to explore the possible expression and regulation of selected $O R$ and TASR genes in human post-mortem orbitofrontal cortex specimens from sporadic $A D$ and non-demented age-matched controls. We focused on early stages of the disease, wherein changes cannot be attributed to neuron loss in comparison to possible alterations occurring with disease progression. Furthermore, we gained insight into an epigenetic gene transcription control mechanism that may underlie etiopathogenetic processes in early $A D$.

\section{Results}

\section{OR and TAS2R genes are expressed in the OFC}

Considering the large number of protein-coding $O R$ and TASR genes in the human genome, ten different genes were selected for this study. Olfactory receptors OR2K2, OR2H2, OR1L8, OR13A1, and OR7A17 genes were selected based on gene families and chromosomal distribution: members of the same gene family (OR2), members of different families (OR1, OR2, OR7, OR13), genes located within the same chromosome (OR2K2 on 9q31.3, OR1L8 on 9q33.2) and genes located in different chromosomes (OR2H2 on 6p22.1, OR13A1 on 10q11.21, OR7A17 on 19p13.12). Bitter taste receptors TAS2R14 and TAS2R5 were selected based on number of known ligands: TAS2R14 is the most broadly tuned human TAS2R with 151 known agonists, and TAS2R5 is one of the most narrowly tuned TAS2R, with only 6 known agonists (BitterDB database) ${ }^{17}$. An ideogram of the distribution of the selected $O R$ and TASR genes on the human genome is presented in Figure $1 \mathrm{a}$.

We first explored whether the selected $O R$ and TASR genes were expressed in OFC and whether they evidenced sexual dimorphism. The transcriptomic analysis performed on non-demented control samples revealed that olfactory receptors $O R 2 K 2, O R 2 H 2$ and $O R 1 L 8$ (Figure 1b) and bitter taste receptors TAS2R5 and TAS2R14 (Figure 1c) are expressed on OFC specimens. No sex differences were observed in the mRNA relative levels of $O R 2 H 2, O R 2 K 2$ and TAS2R14. In contrast, OR1L8 and TAS2R5 were found to be 
significantly higher expressed in female in comparison to male group $(P=0.004$ and $P=0.0002$, respectively) - Figure $1 \mathrm{~b}$ and $1 \mathrm{c}$. For this reason, further analysis of these two transcripts were separated by sex.

OR13A1 expression levels were found to be very low and excessively close to the technique's reliable detection limit, therefore no quantitative analysis was performed on this transcript. No detection was remarked for OR7A17 and for type 1 taste receptors (TAS1R1, TAS1R2, TAS1R3).

\section{Selected OR and TAS2R genes are downregulated in the OFC at early stages of AD}

We next examined $O R$ and TAS2R expression in OFC specimens from Braak I to $V$ stages of sporadic AD and compared to age-matched non-demented controls. We found that $O R$ and TAS2R are markedly downregulated both in male and females, at several stages - Figure 2. With the exception of $O R 2 H 2$ and OR1L8 (female group), that despite displaying a similar tendency did not reach statistical significance, all other transcripts analyzed showed a clear and statistically significant downregulation in Braak I stage compared to non-demented controls, and in some cases also in stage II.

In detail, significant downregulation was observed in OR2K2 at Braak I $(P<0.0001$, Figure 2a), in OR1L8 (male group) at Braak I/II ( $P=0.0071$, Figure 2c), in TAS2R14 at Braak I $(P=0.0017$, Figure $2 \mathrm{~d})$, and in TAS2R5, both groups, at Braak I/II ( $P=0.0114$ and $P=0.0473$, respectively, Figure 2e). Likewise, significant downregulation was also observed in OR2K2 at Braak V $(P=0.0031$, Figure 2a), in OR1L8 (male group) at Braak III/IV ( $P=0.0393$, Figure 2c), in TAS2R14 at Braak IV $(P=0.0225)$ and at Braak V $(P$ $<0.0001$, Figure 2d), and in TAS2R5 in male group at Braak IV/V $(P=0.0137)$, and in female group, at Braak III ( $P=0.0290$, Figure 2e). In the case of OR2K2 and TAS2R14 and TAS2R5 (male group), it is noticeable some attempt of compensation in midst stages, albeit expression levels never reached those of non-demented controls (Figure 2a, 2d and 2e). On the other hand, OR2H2 and OR1L8 expression levels seemed to be restored at advanced stages (Figure $2 \mathrm{~b}$ and $2 \mathrm{c}$ ).

Considering that gene expression was analyzed in post-mortem human brain samples, three different stable housekeeping genes were used for normalization of RT-qPCR data: Glyceraldehyde-3-Phosphate Dehydrogenase (GAPDH), Phosphoglycerate Kinase 1 (PGK1), and Actin Beta (ACTB). No differences were observed in the expression levels of these genes, when comparing age-matched non-demented controls and the different Braak stages. Furthermore, normalization of the target genes with the three independent housekeeping genes produced similar results (data not shown).

Taken together, these findings indicate that selected $O R$ and TAS2R transcripts are expressed in the OFC and differentially downregulated since early stages of sporadic AD. Furthermore, deregulation does not follow disease progression pattern, but rather point toward an epigenetic mechanism that may play a direct role at early stages of $A D$.

$\mathrm{H} 3 \mathrm{~K} 9 \mathrm{me} 3$ epigenetic mark is increased in OFC and is enriched at ORs and TAS2Rs proximal promoters at Braak stage I 
Subsequently, we sought to explore a potential regulatory mechanism mediating $O R$ and TAS2R downregulation in human OFC. Thus far, the transcriptional regulation of $O R$ and TASR in humans still represents a fundamental open question. Yet, interestingly, studies in mouse olfactory epithelium have demonstrated that Olfrgene loci reside in inactive heterochromatin characterized by H3K9me3 and other inhibitory histone methylation marks ${ }^{18}$. Based on this evidence, we initially measured the amount of global H3K9me3 levels in histone extracts of OFC specimens. We found a significant increase in the amount of this repressive signature at Braak I $(P=0.0012)$, and also Braak stages III $(P=0.0007)$, and IV/V $(P=0.0003)$, but surprisingly not at stage II - Figure 3 .

Then we sought to determine whether this inhibitory epigenetic mark was regulating the selected $O R$ and $T A S 2 R$ mRNA levels, focusing in possible alterations at early and late stages of AD (Braak I and IV/V). However, depending on its localization, the $\mathrm{H} 3 \mathrm{~K} 9 \mathrm{me} 3$ histone modification may have different effects on gene expression regulation. While $\mathrm{H} 3 \mathrm{~K} 9 \mathrm{me} 3$ is often found at promoter regions where it represses transcription ${ }^{19-21}$, it may also act as a repressive modification on coding regions ${ }^{22,23}$. Additionally, H3K9me3 can also modulate alternative splicing patterns ${ }^{24-26}$, and in fact, it has been shown that nucleosomes are preferentially positioned over exons and that certain histone modifications show exonic enrichment with effects in splicing regulation ${ }^{27-31}$. Based on these rationales, we designed and performed N-ChIP-qPCR assay to interrogate both H3K9me3-mediated transcriptional repression at each proximal promoter and $\mathrm{H} 3 \mathrm{~K} 9 \mathrm{me} 3$-mediated alternative splicing at the coding region of the candidate genes (Supplementary Figure 1). We found that H3K9me3 epigenetic mark is indeed particularly enriched at $O R$ and TAS2R proximal promoter at Braak stage I - Figure 4a. In detail, is significantly enriched at OR2H2 $(P=0.0031), O R 2 K 2(P=0.0003)$ and OR1L8 $(P=0.0002)$, and t TAS2R14 $(P=0.0006)$ and TAS2R5 $(P=0.0041)$ proximal promoters at Braak stage $I$ in comparison to age-matched non-demented control group.

In contrast, we found no enrichment on coding regions. Except for $\mathrm{OR} 2 \mathrm{H} 2$, all the other targets showed considerably lesser or no significant difference in the percentages of the input chromatin precipitated between Braak stage I and non-demented control groups - Figure 4b. In detail, enrichment of the H3K9me3 repressive mark was only found on OR2H2 $(P=0.0033)$ and on TAS2R5 $(P=0.0172)$ coding regions at Braak stage I.

On the other hand, the $\mathrm{H} 3 \mathrm{~K} 9 \mathrm{me} 3$ repressive mark was found to be markedly reduced with disease progression (Braak stages IV/V) both in the proximal promoter and the coding region of all the targets analyzed - Figure $4 \mathrm{a}$ and $4 \mathrm{~b}$. This marked reduction in advanced stages of the disease was noticed both in comparison with Braak stage I and with non-demented control group, although the loss of the H3K9me3 repressive mark was evidently more pronounced in comparison with the incipient stages of the disease. In detail, OR2H2 $(P<0.0001$ and $P=0.0025)$, OR2K2 $(P<0.0001$ and $P=0.0165), O R 1 \angle 8(P<$ 0.0001 and $P=0.0057)$, TAS2R14 $(P<0.0001$ and $P=0.0279)$ and TAS2R5 $(P<0.0001$ and $P=0.0019)$ proximal promoters presented a prominent reduction in the H3K9me3 mark at Braak stages IV/V in comparison with Braak stage I and with non-demented control group, respectively - Figure 4a. Likewise, OR2H2 $(P=0.0003$ and $P=0.0106), O R 2 K 2(P=0.0002$ and $P=0.0019), O R 1 L 8(P=0.0002$ and $P=$ 
0.0172), TAS2R14 $(P<0.0001$ and $P=0.0004)$ and TAS2R5 $(P=0.0003$ and $P=0.0244)$ coding regions presented a pronounced decrease in the H3K9me3 mark at Braak stages IV/V in comparison with Braak stage I and with non-demented control group, respectively - Figure 4b.

Taken together, these data suggest that at early stages of sporadic $A D$, the prominent enrichment of the H3K9me3 repressive mark in the proximal promoter of the selected chemoreceptor genes could be mediating their downregulation. On the other hand, with disease progression, the reduction on the $\mathrm{H} 3 \mathrm{~K} 9 \mathrm{me} 3$ repressive mark observed in both promoter and coding regions, suggests that the inhibitory function associated with $\mathrm{H} 3 \mathrm{~K} 9 \mathrm{me} 3$ is not required and that an alternative repression mechanism might be involved.

\section{Identification of $\mathrm{H} 3 \mathrm{~K} 9 \mathrm{me} 3$ interactome in OFC at early and advanced stages of $\mathrm{AD}$}

To further inquire the mechanism involved in $O R$ and TAS2R downregulation by H3K9me3 repressive mark at early Braak stages, we performed mass spectrometry-based proteomics to identify potential differences in the H3K9me3 interactome in OFC between early and advanced stages. Bona fide H3K9me3-binding partners were distinguished from background, nonspecific lgG-associated proteins (rabbit IgG IP), by eliminating mutual proteins, including common contaminants such as immunoglobulins and keratins. A total of 107 unique proteins were identified. A gene ontology enrichment analysis on biological process was performed with Phanter ${ }^{32}$ (Figure $5 \mathrm{a}-\mathrm{c}$ ). A total of 5 genes were uniquely mapped to Braak I, 13 genes were common to Braak I and V, and 22 genes unique to Braak $V$. Significant differences were found in fold enrichment for genes common to Braak I and V (Supplementary table 3), and also genes unique to Braak V (Supplementary table 4), but not in Braak I. At both Braak stages, the highest fold enrichment was observed in chromatin silencing and nucleosome assembly process, whereas at Braak $\mathrm{V}$ it included cytoplasmic translation and regulation of mRNA splicing.

We then focused in candidate $\mathrm{H} 3 \mathrm{~K} 9$ me3-interacting proteins exclusively identified at early stages and not in advanced $A D$ wherein many confounding factors might be in play. After filtering for proteins uniquely identified at Braak I and not Braak V, a total of 7 proteins were identified and Methyl CpG-binding protein 2 (MeCP2), a DNA metabolism protein, was pinpointed as a candidate interactor of $\mathrm{H} 3 \mathrm{~K} 9 \mathrm{me} 3$ in Braak I that could mediate $O R$ and $T A S 2 R$ regulation. MeCP2 is a highly abundant chromosomal protein within the brain, particularly in neurons, and it plays a multifaceted role in gene expression regulation and chromatin organization. MeCP2 can function as a transcriptional repressor that targets methylated DNA, but it has also been shown to interact with methylated histone proteins ${ }^{33-35}$.

To validate the identified $\mathrm{H} 3 \mathrm{~K} 9$ me3-MeCP2 interaction, we then performed reciprocal coimmunoprecipitations in nuclear extracts. Both $\mathrm{H} 3 \mathrm{~K} 9 \mathrm{me} 3$ and MeCP2 were detected in the reciprocal CoIP eluates and were absent from the IgG control immunoprecipitation - Figure $5 \mathrm{~d}$.

Additionally, we sought to investigate if there were alterations in the global levels of MeCP2 protein at early (Braak I) and advanced stages (Braak V) in comparison to non-demented controls. We found a 
significant increase of MeCP2 levels at both Braak I $(P=0.0050)$ and Braak V $(P=0.0089)$ - Figure 5e-g. No significant sex differences were observed.

These findings suggest that $\mathrm{H} 3 \mathrm{~K} 9 \mathrm{me} 3-\mathrm{MeCP} 2$ interaction is an early event in sporadic $A D$, possibly regulating different genes that could be involved in the pathogenesis of the disease. In particular, MeCP2 might be implicated in $O R$ and $T A S 2 R$ genes expression regulation through interaction with $\mathrm{H} 3 \mathrm{~K} 9 \mathrm{me} 3$ in early stages of $A D$.

\section{Discussion}

Emerging evidence indicates that brain expression of some $O R$ and TAS2R genes is modulated in neurological diseases ${ }^{8-11}$. In AD, for instance, a small subset of mRNAs was found to be deregulated (either up- or down-regulated) in human entorhinal cortex and frontal cortex area 8, albeit exclusively in limbic and isocortical stages where many confounding factors might be in play. In frontal cortex area 8 , specifically, only ORAF4 and ORS2L 1 were found to be up-regulated, and no alteration was observed in TAS2Rs mRNA expression at any stage ${ }^{10}$.

In our study, we sought to explore the possible expression and regulation of selected $O R$ and TASR mRNAs in human OFC (areas 10,11 and 47), a polymodal region of the frontal cortex with great anatomical connectivity to sensory areas and limbic structures involved in emotion and memory. The human genome contains an extensive number of protein-coding $O R$ and TAS2R genes, thus the present study was not intended to provide a comprehensive list of deregulated mRNAs. We found OFC expression of some $O R$ and TAS2R mRNAs both in male and female samples, most at comparable levels, and expression of a subset of sexually dimorphic $O R$ and TAS $2 R$ mRNAs. These findings indicate sex-specific vulnerability of certain $O R$ and $T A S 2 R$ genes. Although steroid hormones such as androstenone and androstadienone have been identified as ORs ligands ${ }^{36}$, it is unlikely that steroid hormones or their derivatives play a major role in modulation of these genes sex-specific vulnerability in this particular setting, considering the frequent age- and AD-related decline in circulating and brain levels of sex steroid hormones ${ }^{37}$. Thus, at least a subset of these chemoreceptor genes might be modulated by distinct sexually-dimorphic ligands or signals.

On the other hand, we were not able to detect expression of type 1 taste receptors (TAS1R1, TAS1R2, TAS1R3) mRNAs in OFC specimens by RT-qPCR. Although Tas1r expression was described in rodent brain 14,15, and cDNA microarray and RNA sequencing data supported the idea of TAS1R expression in multiple human brain regions, particularly in limbic system areas ${ }^{38}$, no study thus far has validated the expression of TAS1R mRNAs in any region of the human brain, which overall indicates that humans most likely lack TAS1Rs expression in OFC. Notwithstanding, considering their proposed involvement in the maintenance of glucose homeostasis in mouse hypothalamic cells ${ }^{15}$, and that impaired cerebral glucose metabolism is an invariant pathological feature of $A D$, comprehensive assessment of TAS1R expression in human brain would be an important topic for future studies. 
Subsequently, we found that $O R s$ and TAS2Rs mRNA expression levels are markedly downregulated in AD specimens, both in male and female samples, particularly in its most incipient stage, Braak stage $\mathrm{I}$, and in most cases also in advanced stages. These findings indicate that transcripts deregulation does not follow a disease progression pattern, but rather points toward a regulation mechanism that may play a direct role in incipient $A D$. Also, these alterations do not appear to be associated with neuronal loss or to be a mere outcome of OFC neurodegeneration, as the appearance of AD-associated neuropathology in the OFC is preceded by a decline in these transcripts mRNA levels at incipient stages wherein neurofibrillary tangle involvement is still limited to trans-entorhinal and entorhinal cortices. Furthermore, in some cases their expression levels seem to be restored at advanced stages wherein neurofibrillary tangles are at that point extensive in the neocortical regions.

In contrast to previous observations, wherein $O R$ and TAS2R expression was mainly found to be upregulated or unchanged in entorhinal and frontal cortex, and alterations were limited to more advanced stages of $A D{ }^{10}$, it is puzzling to remark that in our study, we found downregulation in OFC already at early stages. Although dissimilarities can be attributed in part to different $O R s$ being analyzed, and therefore to receptor-specific vulnerability, two common TAS2Rs (TAS2R5, TAS2R14) were investigated, indicating a possible cerebral cortex region-specific vulnerability to $O R$ s and TAS2Rs transcriptional changes.

Beyond cerebral $O R$ and TA2SR mRNAs expression deregulation (manifested either as up- or downregulation) documented in neurological disorders ${ }^{8-11}$, there is limited knowledge regarding their involvement on physiological or pathological processes in neuronal systems. Nevertheless, the characterization of their physiological roles in other non-chemosensory tissues and their involvement in similar pathological settings suggest that these chemoreceptors might have a unique functionality in these systems.

It has been shown that TAS2Rs act as immune sentinels, mobilizing defense mechanisms against pathogenic aggression. For instance, these receptors are stimulated in the presence of gram-negative quorum-sensing molecules, leading to increased nitric oxide production and mucociliary clearance activation during upper airways inflammation ${ }^{39}$. In contrast, recent evidence has shown that downregulation of key components of the taste signaling cascade is associated with increased production of pro-inflammatory mediators and oxidative stress molecules in diabetic nephropathy 40 , which initiate inflammatory responses similar to those observed in neurological diseases. In this sense, although there is no evidence so far on the involvement of TAS2Rs in neuroinflammatory responses, it is plausible that the downregulation of cerebral TAS2R mRNAs, previous to the appearance of ADassociated neuropathology in the OFC, could be associated with a possible deficiency on the response to toxigenic substances/neurotoxins and microbial components, which under normal conditions could be necessary to prevent their pathogenic action in the cerebral microenvironment, and consequently necessary to prevent neuroinflammatory reactions. This might represent an important etiopathogenetic mechanism, notwithstanding additional comprehensive studies are required to understand the potential 
molecular mechanisms connecting TAS2Rs-mediated surveillance of the extracellular milieu and neuroinflammation.

Reports on the potential ORs involvement on neuropathological processes have also started to emerge. Remarkably, it has been shown that the activation by acetophenone of overexpressed human OR4M1 in mouse primary cortico-hippocampal neurons could lead to attenuation of abnormal microtubuleassociated tau protein phosphorylation via a JNK signaling pathway ${ }^{41}$. These findings suggest that ORs may interfere with aberrant tau hyperphosphorylation, which is one of the pathological hallmarks of $A D$, and that ligand-induced activation of ORs might result in protection against tau neuropathological features. Therefore, it is plausible that decreased expression of $O R$ transcripts, as documented in our study, might be associated with AD-related tauopathy. Nonetheless, additional studies are required to dissect in detail the involvement of cerebral ORs in the attenuation of tau neuropathology.

On the other hand, the transcriptional control mechanisms responsible for $O R$ and TAS2R genes regulation in humans still remains elusive. In mouse olfactory epithelium, repression of Olfrgenes has been associated with $\mathrm{H} 3 \mathrm{~K} 9 \mathrm{me} 3$ and another inhibitory histone methylation mark, $\mathrm{H} 4 \mathrm{~K} 2 \mathrm{Ome} 3{ }^{18}$. The presence of $\mathrm{H} 3 \mathrm{~K} 27 \mathrm{me}$, another inhibitory histone methylation mark, over gene promoters has also been highly correlated with gene repression. Yet, it has been shown that promoters marked by H3K27me3 remain accessible to binding by general transcription factors and paused RNA polymerase ${ }^{42,43}$, while chromatin marked by $\mathrm{H} 3 \mathrm{~K} 9 \mathrm{me} 3$, instead, occludes transcription factors binding with diverse DNA-binding domains ${ }^{44}$.

Despite the assumption that the mechanisms regulating $O R$ expression outside of the olfactory epithelium could be markedly different, it is enigmatic whether this heterochromatic silencing mechanism is implemented also in human tissues in physiological or pathological settings.

Therefore, we sought to investigate the potential alterations on $\mathrm{H} 3 \mathrm{~K} 9 \mathrm{me} 3$ in human OFC in sporadic AD. Remarkably, we found a significant increase of H3K9me3 global levels at both early and advanced stages of sporadic AD. These findings suggest that a gain of specific histone methyltransferases or loss of specific histone demethylases function might be mediating the upsurge of $\mathrm{H} 3 \mathrm{~K} 9 \mathrm{me} 3 \mathrm{global}$ levels observed at early stages of the disease and could be associated with the $O R$ and TAS2Rs genes repression. This epigenetic alteration can also be associated with the repression of a number of ADrelated genes, such as neuronal activity-related genes, $A \beta$ clearance or production machinery genes, and tau-related genes, which makes the scenario a subject of profound interest. Thus far, the study of the potential alterations on $\mathrm{H} 3 \mathrm{~K} 9$ methylation in $A D$ has been inconclusive as only a few studies have addressed this question. Increased global H3K9me2 levels were reported in human occipital and prefrontal (area 10) cortex ${ }^{45,46}$ and H3K9me3 in temporal cortex ${ }^{47}$, whereas reduced H3K9me2 levels were observed in hippocampal neurons ${ }^{48,49}$. Likewise, the potential alterations on $\mathrm{H} 3 \mathrm{~K} 9$ methylation at gene-specific locus in AD has only been explored in a familial AD mouse model, wherein H3K9me2 enrichment was found at glutamate-receptor genes promoter in prefrontal cortex ${ }^{46}$. Interestingly, 
integrated analysis of genome-wide ChIP- and mRNA-sequencing data showed H3K9me3 promoter occupancy in synaptic function-related genes in human temporal cortex in advanced stages of $A D 47$.

Based on the increase of $\mathrm{H} 3 \mathrm{~K} 9 \mathrm{me} 3$ global levels we observed in OFC and the effects that H3K9me3 may entail depending on the specific genomic loci, we interrogated both $\mathrm{H} 3 \mathrm{~K} 9 \mathrm{me}$-mediated transcriptional repression and $\mathrm{H} 3 \mathrm{~K} 9$ me3-mediated alternative splicing at the proximal promoter and the coding region of the candidate $O R$ and $T A S 2 R$ genes. We focused on possible alterations at early stages in which changes cannot be attributed to AD-derived neuronal loss in comparison with possible changes occurring with disease progression. We found a prominent enrichment of the H3K9me3 repressive mark in the proximal promoter of the target chemoreceptor genes at Braak stage I, which suggests that an H3K9me3-mediated mechanism could be responsible for $O R s$ and TAS2Rs transcriptional control in this brain region. In addition, these findings raise the possibility that this silencing mechanism might also be implemented in other non-chemosensory tissues and organs and in other physiological and pathological contexts. On the other hand, at later stages, we found a pronounced reduction of the H3K9me3 repressive mark in both promoter and coding regions, suggesting that the inhibitory function associated with $\mathrm{H} 3 \mathrm{~K} 9 \mathrm{me} 3$ is not required at these sites and that an alternative repression mechanism might be involved at advanced stages.

There are, however, some hindrances in understanding the transcriptional control of these genes expression due to the lack of a complete characterization of $O R$ promoters and transcription start sites (TSS). The analysis of each proximal promoter regulatory region was based on a consensus localization of transcription factor binding sites clustered at a distance of 100 to $300 \mathrm{bp}$ from the TSS reported on the upstream regions of a number of human $O R$ genes ${ }^{50}$. We cannot exclude that transcription regulation might be controlled by different regulatory sites or the presence of different promoters for the selected $O R$ and TAS2R genes.

Afterwards, we inquired the potential differences in the H3K9me3 interactome in OFC between early and advanced stages by mass spectrometry-based proteomics, and the analysis pinpointed MeCP2 as a candidate interactor of H3K9me3 in Braak stage I. In fact, in addition to methylated DNA, MeCP2 has been shown to interact with specific histone methylation marks. MeCP2 has been shown to bind to H3K9me2 and H3K27me3 nucleosomes in mouse brain nuclear extracts ${ }^{33}$, and was found to be associated with $\mathrm{H} 3 \mathrm{~K} 9 \mathrm{me} 2$ in the $/ \mathrm{L}-6$ gene upstream region in pancreatic adenocarcinoma cell lines ${ }^{34}$. Recently, it has also been shown that MeCP2 can regulate gene expression through recognition of H3K27me3 and that MeCP2-H3K27me3 interaction is independent of DNA methylation ${ }^{35}$.

We then sought to investigate the potential alterations on MeCP2 protein levels in the human OFC and we found a pronounced increase in this protein expression already at early stages of the disease. Alterations in the homeostatic levels of this protein at this early stage could have important functional consequences for AD pathogenesis. Further validation of H3K9me3-MeCP2 interaction at early stages of AD by reciprocal co-IPs puts forwards this epigenetic repressive mechanism as an early event in AD that could be implicated in the transcriptional regulation of $O R$ and TAS2R genes and other AD-related genes. 
Validation of H3K9me3-MeCP2 interaction was just the first step in elucidating this mechanism, notwithstanding, tissue amount requirements and differences in antibody efficiency have hindered further analysis on the co-enrichment of these two chromatin-associated proteins on the selected $O R$ and TAS2R proximal promoters by sequential ChIP.

In summary, our findings indicate that the upsurge in $\mathrm{H} 3 \mathrm{~K} 9 \mathrm{me} 3$ and MeCP2 proteins and epigenetic repression of chemoreceptor genes are early events in $A D$ pathogenesis, as these alterations seem to precede more conventional pathological features such as amyloid plaque formation and tauopathy. It is possible that $O R$ and $T A S 2 R$ genes and other AD-related genes become inactive through $\mathrm{H} 3 \mathrm{~K} 9 \mathrm{me} 3$ engagement in early stages of $A D$, and as the disease progresses, the reversible relaxation of H3K9me3enriched chromatin may be compromised and lead to the constitutive silencing of these genes. Therefore, it will be necessary to further investigate the mechanisms responsible for $\mathrm{H} 3 \mathrm{~K} 9 \mathrm{me} 3$ increase in early stages of $A D$ and whether $\mathrm{H} 3 \mathrm{~K} 9 \mathrm{me}$-enriched chromatin and $\mathrm{H} 3 \mathrm{~K} 9 \mathrm{me}-\mathrm{landscaped}$ genes can be reversibly modulated.

\section{Methods}

\section{Human post-mortem brain tissue samples}

Human post-mortem specimens of orbitofrontal cortex (Brodmann's area 10, 11 and 47) from twenty-five age-matched non-demented controls and forty-nine AD Braak I-V cases were obtained from the Institute of Neuropathology Brain Bank (HUB-ICO-IDIBELL Biobank, Barcelona, Spain), the Basque Biobank (País Vasco, Spain), and the Hospital 12 Octubre Biobank (i+12 Biobank, Madrid, Spain). All samples were acquired following the relevant guidelines and regulations approved by the local ethics committee from the Hospital 12 de Octubre Research Institute, and by institutional ethic committees from the Institute of Neuropathology Brain Bank, the Basque Biobank, and the Hospital 12 de Octubre Biobank. Samples were dissected at the time of autopsy with the informed consent of donors or their legal next of kin and immediately frozen and stored at $-80^{\circ} \mathrm{C}$ until use. All samples were anonymized with regard to their personal data. The post-mortem interval between death and tissue processing was between 1 and 24 hours in all cases. The neuropathological diagnosis of sporadic AD was based on the NFT pathology classification according to the Braak and Braak nomenclature ${ }^{2,3}$. Demographic and tissue-related features of OFC specimens are summed up in Table 1 and detailed in Supplementary Table 1. 
Table 1

Demographic and tissue-related features of orbitofrontal cortex specimens $(n=74)$.

\begin{tabular}{|c|c|c|c|c|c|c|c|}
\hline Sex & $\mathbf{n}$ & $\begin{array}{l}\text { Age at death } \\
\text { (years) }\end{array}$ & SEM & $\begin{array}{l}\text { PMI } \\
\text { (hours) }\end{array}$ & SEM & APOE Genotype (n) & $\begin{array}{l}\text { Braak } \\
\text { Stage }\end{array}$ \\
\hline Male & 13 & 65.5 & 4.1 & 11.3 & 2.7 & $3 / 3(8), 2 / 3(1), 3 / 4(1)$ & \multirow[t]{2}{*}{0} \\
\hline Female & 12 & 73.7 & 4.9 & 10.3 & 2.2 & $3 / 3(8), 2 / 3(1), 3 / 4(1)$ & \\
\hline Male & 9 & 64.3 & 2.0 & 6.1 & 0.8 & $3 / 3(5), 2 / 3(2), 3 / 4(2)$ & \multirow[t]{2}{*}{1} \\
\hline Female & 6 & 69.0 & 3.9 & 7.9 & 1.9 & $3 / 3(3), 2 / 2(1), 3 / 4(2)$ & \\
\hline Male & 8 & 71.1 & 3.8 & 5.3 & 0.8 & $3 / 3(6), 2 / 3(2)$ & \multirow[t]{2}{*}{ II } \\
\hline Female & 3 & 65.3 & 3.8 & 6.4 & 2.1 & $3 / 3(3)$ & \\
\hline Male & 5 & 73.2 & 3.8 & 5.2 & 0.5 & $3 / 3(3), 3 / 4(2)$ & \multirow[t]{2}{*}{ III } \\
\hline Female & 5 & 81.8 & 2.8 & 6.5 & 2.0 & $3 / 3(4), 3 / 4(1)$ & \\
\hline Male & 2 & 79.0 & 5.0 & 4.9 & 0.1 & $3 / 3(2)$ & \multirow[t]{2}{*}{ IV } \\
\hline Female & 2 & 89.0 & 1.0 & 7.0 & 2.9 & $3 / 3(2)$ & \\
\hline Male & 6 & 79.8 & 2.9 & 7.7 & 2.1 & $3 / 3(3), 3 / 4(3)$ & \multirow[t]{2}{*}{ V } \\
\hline Female & 3 & 76.3 & 2.3 & 5.0 & 0.4 & $3 / 3(1), 2 / 3(1), 3 / 4(1)$ & \\
\hline TOTAL & 74 & 71.8 & 1.4 & 7.8 & 0.7 & $\begin{array}{l}3 / 3(48), 2 / 2(1), 2 / 3(7) \\
3 / 4(13)\end{array}$ & \\
\hline
\end{tabular}

\section{Genomic DNA extraction and APOE genotype}

Genomic DNA extraction was carried out with the DNeasy Blood and Tissue Kit (Qiagen, Hilden, Germany) according to manufacturer's instructions. DNA yields and $\mathrm{A}_{260}$ ratios were determined with a NanoDrop ND-1000 spectrophotometer (NanoDrop Technologies, DE, USA). Identification of $A P O E \in 2, \epsilon 3$, and $€ 4$ alleles was performed by taqman assays using the LightMix ApoE C112R and R158C Kit from TibMolbiol (Roche Diagnostics, BE, Germany) to identify the ApoE missense variants R158C (rs7412) and C112R (rs429358), on a LightCycler 480 II Instrument (Roche, IN, USA).

\section{RNA isolation and real-time quantitative RT-PCR}

Total RNA was isolated using Trizol Reagent (Life Technologies, CA, USA) according to the manufacturer's procedure. RNA yields and $A_{260}$ ratios were determined by spectrophotometry. The RNA isolation was followed by DNase treatment (TURBO DNA-free DNase treatment \& Removal Reagents, Ambion, CA, USA) for removal of genomic DNA. 
Each sample was reverse transcribed using $0.75 \mu \mathrm{g}$ of purified total RNA, a mixture of oligo(dT) and random hexamer primers and $\mathrm{RNase} \mathrm{H}+$ iScript reverse transcriptase (iScript cDNA synthesis kit, Bio-Rad, CA, USA). Transcript relative quantifications were performed on a LightCycler 480 II Instrument (Roche, IN, USA) in 384-well plates. Each real-time quantitative PCR reaction was run in triplicate. Primer sequences are listed in Supplementary Table 2. GAPDH, ACTB and PGK1 were used as housekeeping genes. The fold difference in expression of the genes of interest between non-demented controls $(n=25)$ and AD Braak I$V$ cases $(n=49)$ was calculated by the $2^{-\Delta \Delta C t}$ method ${ }^{51}$.

\section{Total histone extraction and measurement of global histone H3 lysine 9 tri-methylation}

Total histones extracts were isolated from non-demented controls $(n=24)$ and AD Braak I-V cases $(n=49)$ using the EpiQuik total histone extraction kit (Epigentek, NY, USA) according to the manufacturer's instructions. Protein concentrations were quantitated using the Pierce BCA protein assay kit (Thermo Scientific, IL, USA). H3K9me3 amounts were measured with the EpiQuik global tri-methyl histone H3K9 quantification kit (Epigentek, NY, USA), according to the manufacturer's protocols.

\section{Native chromatin immunoprecipitation (N-ChIP) and quantitative real-time PCR analysis}

Native chromatin immunoprecipitation (N-ChIP) was performed according to Donovan \& Lichota ${ }^{52}$ with slight modifications. Isolation of total chromatin was carried out on OFC specimens from age-matched controls $(n=15), A D$ Braak I $(n=14)$ and Braak IV-V samples $(n=10)$. Chromatin fragmentation was achieved by enzymatic digestion with micrococcal nuclease (ThermoFisher, IL, USA) and micro dialysis was performed in micro float-A-Lyzer 8-10 kDa pore width devices (SpectrumLabs, CA, USA).

For immunoprecipitation (IP) of the resulting chromatin fragments, $3 \mu \mathrm{g}$ of ChIP-grade anti-H3K9me3 antibody (ab8898, Abcam, MA, USA) were used. No antibody was added to the negative control (mock). A fraction of the chromatin samples was reserved as input. IP samples were incubated with protein Acoated magnetic beads (Dynabeads Protein A, Invitrogen, CA, USA) and the immune complexes eluted from beads. Histones proteins were removed by proteinase $K$ digestion (Roche diagnostics, MA, Germany) and DNA purification was achieved with a spin column-based Speedtools PCR clean-up kit (Biotools, B\&M Labs, Madrid, Spain).

To examine H3K9me3 binding at each chemoreceptor locus, quantitative real-time PCR was performed on the precipitated DNA fragments using two pairs of oligonucleotide primers designed to produce amplicons within each chemoreceptor proximal promoter (-100bp to -300bp upstream of the transcription starting site) and coding regions (primers used in the transcriptomic analysis). For details on primers positions and sequences, see Supplementary Figure 1 and Supplementary Table 2. Each reaction was run in triplicate in a LightCycler 480 II Instrument (Roche, IN, USA) in 384-well plates. Results are reported as the percentage of the input chromatin that is precipitated at each region of interest.

Native nuclear complex co-immunoprecipitation and reverse phase-liquid chromatography coupled to mass spectrometry (RP-LC-MS/MS) analysis 
To investigate the potential interactome of the repressive histone mark H3K9me3 in OFC specimens, native nuclear complex co-immunoprecipitation (Co-IP) was combined with reverse phase-liquid chromatography coupled to mass spectrometry (RP-LC-MS/MS) analysis.

Nuclear extracts from pooled male Braak I and Braak V (three specimens per pool) were prepared as follows. Hypotonic buffer and NP-40 detergent was used to separate the cytoplasmic fraction, nuclei were lysed and nuclear proteins were recovered in low-salt buffer in the presence of protease inhibitors cocktail. DNA digestion by an enzymatic shearing enzyme (MNase, Thermo Fisher, IL, USA) was performed to release un-dissociated protein complexes from the DNA. Total protein concentrations were quantitated using the Pierce BCA protein assay kit (Thermo Scientific, IL, USA).

Enrichment of the H3K9me3 target was performed by combining $1 \mathrm{mg}$ of each protein-complexes fraction with $12 \mu \mathrm{g}$ of ChIP-grade anti-H3K9me3 recombinant antibody (ab176916, Abcam, MA, USA). A negative control was set up with pooled protein-complexes fractions and $12 \mu \mathrm{g}$ of rabbit lgG (I5006, Sigma-Aldrich, MO, USA). Co-IP reactions were then incubated with protein A-coated magnetic beads (Dynabeads Protein A, Invitrogen, CA, USA) and protein-complexes were eluted from beads.

After in-gel chymotrypsin digestion of protein-complexes, the desalted peptides were analyzed by RP-LCMS/MS in an Easy-nLC II system coupled to an ion trap LTQ Orbitrap Velos Pro hybrid mass spectrometer (Thermo Scientific, MA, USA). Peptides were on-line concentrated by reverse phase chromatography in a $0.1 \mathrm{~mm} \times 20 \mathrm{~mm}$ C18 RP precolumn (Thermo Scientific, MA, USA), and then separated in a $0.075 \mathrm{~mm} \mathrm{x}$ $250 \mathrm{~mm}$ C18 RP column (Thermo Scientific, MA, USA) operating at $0.3 \mu \mathrm{l} / \mathrm{min}$. Peptides were eluted using a 90-min dual gradient and electrospray ionization (ESI) was performed using a nano-bore stainless steel emitter $30 \mu \mathrm{m}$ ID (Proxeon, ODE, Denmark) interface at $2.1 \mathrm{kV}$ spray voltage with S-Lens of $60 \%$.

Peptides were detected in survey scans from 400 to 1600 amu (1 $\mu$ scan), with Orbitrap resolution set at 30.000 , followed by 20 data-dependent MS/MS scans (Top 20), using an isolation width of $2 \mathrm{u}$ (in massto-charge ratio units), normalized collision energy of 35\%, and dynamic exclusion applied during $60 \mathrm{~s}$ periods. Charge-state screening was enabled to reject unassigned and singly charged protonated ions. The mass spectrometer was further operated in the selected MS/MS ion monitoring (SMIM) mode ${ }^{53}$, wherein the LTQ-Orbitrap-Velos-Pro detector was programmed to perform, along the same entire gradient, a continuous sequential operation in the MS/MS mode on the doubly or triply charged ions corresponding to histone H3.1 peptides previously selected from the theoretical prediction.

Peptide identification from MS/MS raw data files was carried out using PEAKS Studio $X$ search engine (Bioinformatics Solutions Inc., ON, Canada) and database search was performed against Uniprot Homo sapiens.fasta (71,768 entries, UniProt) (decoy-fusion database). Searching parameters were selected as follows: chymotrypsin cleavage after tyrosine, tryptophan, phenylalanine and leucine (semi-specific), with up to two missed cleavage sites, variable modifications of methionine oxidation, cysteine carbamidomethylation, lysine and arginine methylation and lysine acetylation, and mass tolerances of 20 ppm and 0.6 Da for precursor and fragment ions, respectively. Maximum false discovery rate (FDR) for 
peptide spectrum matches were set to 0.01 . For protein identification, a minimum of two distinct peptides and at least one unique sequence were set as the threshold for successful peptide assignment.

Gene ontology enrichment analysis on biological process for the genes representative of the proteins identified in each dataset was performed with Panther software ${ }^{32}$.

\section{Co-immunoprecipitation and Western blot}

The interaction between $\mathrm{H} 3 \mathrm{~K} 9 \mathrm{me} 3$ and $\mathrm{MeCP} 2$ was validated in native nuclear complex from pooled male Braak I $(n=3)$ cases. Immunoprecipitation was performed with $50 \mu \mathrm{g}$ of the nuclear proteincomplexes fraction and $1 \mu \mathrm{g}$ of the corresponding ChIP-grade antibodies. A negative control was set up with rabbit IgG (15006, Sigma-Aldrich, MO, USA). Western blotting was performed as per standard protocol. The antibodies used were as follows: ChIP-grade rabbit anti-H3K9me3 (ab176916, abcam, MA, USA), 1:1000; ChIP-grade rabbit anti-MeCP2 (C15410052, Diagenode, Liege, Belgium), 1:1000; HRPconjugated goat anti-rabbit (G21234, Invitrogen, OR, USA), 1:5000.

For MeCP2 global levels quantification, nuclear protein-complexes fractions were prepared from OFC specimens from non-demented controls $(n=18)$, Braak I $(n=15)$ and Braak $V(n=9)$ samples as mentioned previously. Specimens from same sex and diagnostic were pooled (two or three per pool whenever possible) to minimize the amount of tissue required per sample. $30 \mu \mathrm{g}$ of nuclear protein-complexes inputs were resolved on 4-20\% Mini-PROTEAN TGX Stain-free Precast gels (Bio-Rad, CA, USA) and stainfree detection was performed in a Gel Doc EZ imaging system (Bio-Rad, CA, USA). Antibodies used were as follows: ChIP-grade rabbit anti-MeCP2 (C15410052, Diagenode, Liege, Belgium), 1:1000; HRPconjugated goat anti-rabbit (G21234, Invitrogen, OR, USA), 1:5000. For total protein normalization, quantification of stain-free total protein was performed using the Image Lab software, version 6.1 (BioRad, CA, USA). A pool of samples was loaded to compare across assays.

\section{Statistical analysis}

Statistical analyses were performed using GraphPad Prism, version 6.0 (GraphPad Software, CA, USA) and IBM SPSS Statistics for Windows, version 20 (IBM Corp., NY, USA). The normality of distribution of the different variables was tested with Kolmogorov-Smirnov and Shapiro-Wilk test and the homogeneity of variance was assessed using Levene's test. Nonparametric Mann-Whitney U test (two-tailed) was used to compare sex differences in transcripts relative expression. Acquired data was compared between $A D$ cases and non-demented specimens using the nonparametric Kruskal-Wallis $\mathrm{H}$ test followed by Dunn's multiple comparisons post-hoc test. The acquired data are presented as mean values \pm standard error of the mean (SEM). $P$ values of less than 0.05 were considered statistically significant.

\section{Declarations}

\section{Acknowledgements}

This research was supported by grants from Instituto de Salud Carlos III (FIS18/00118; FIS21/0063), FEDER, Comunidad de Madrid (S2017/BMD-3700; NEUROMETAB-CM), and CIBERNED (CB07/502). 


\section{Author Contributions}

V.C.A., E.C. and J.F.S. conceived the study. V.C.A and J.F.S designed the study and coordinated experiments and analysis. V.C.A. and J.F.S wrote the original draft. I.F. provided patients specimens. E.C. acquired the financial support. All authors participated in reviewing and editing of the manuscript. All authors read and approved the manuscript.

\section{Competing Interests statement}

The authors declare no competing interests.

\section{Data availability}

All relevant data supporting the key findings of this study are available within the article and its Supplementary Information files or from corresponding authors on request.

\section{References}

1. Querfurth, H. W. \& LaFerla, F. M. Alzheimer's disease: mechanism of disease. The New England Journal of Medicine, 362, 329-344 (2010).

2. Braak, H. \& Braak, E. Neuropathological stageing of Alzheimer-related changes. Acta Neuropathol, 82, 239-259 (1991).

3. Braak, H., Alafuzoff, I., Arzberger, T., Kretzschmar, H. \& Tredici, K. Staging of Alzheimer diseaseassociated neurofibrillary pathology using paraffin sections and immunocytochemistry. Acta Neuropathol, 112, 389-404 (2006).

4. Zhong, S. et al. A single-cell RNA-seq survey of the developmental landscape of the human prefrontal cortex., 555, 524-528 (2018).

5. Nestor, P. G. et al. In search of the functional neuroanatomy of sociality: Mri subdivisions of orbital frontal cortex and social cognition. Social Cognitive and Affective Neuroscience, 8, 460-467 (2013).

6. Catani, M. \& Dell'Acqua, F. Thiebaut de Schotten, M. A revised limbic system model for memory, emotion and behaviour. Neuroscience and Biobehavioral Reviews, 37, 1724-1737 (2013).

7. Pluznick, J. L. \& Caplan, M. J. Novel sensory signaling systems in the kidney. Current Opinion in Nephrology and Hypertension, 21, 404-409 (2012).

8. Garcia-Esparcia, P. et al. Functional genomics reveals dysregulation of cortical olfactory receptors in parkinson disease: Novel putative chemoreceptors in the human brain. Journal of Neuropathology and Experimental Neurology, 72, 524-539 (2013).

9. Grison, A. et al. Mesencephalic dopaminergic neurons express a repertoire of olfactory receptors and respond to odorant-like molecules. BMC Genomics, 15, 729 (2014).

10. Ansoleaga, B. et al. Dysregulation of brain olfactory and taste receptors in AD, PSP and CJD, and ADrelated model., 248, 369-382 (2013). 
11. Ansoleaga, B. et al. Decrease in olfactory and taste receptor expression in the dorsolateral prefrontal cortex in chronic schizophrenia. Journal of Psychiatric Research, 60, 109-116 (2015).

12. Wiener, A., Shudler, M., Levit, A. \& Niv, M. Y. BitterDB: A database of bitter compounds. Nucleic Acids Res, 40, D413-9 (2012).

13. Duarte, A. C. et al. Bitter taste receptors profiling in the human blood-cerebrospinal fluid-barrier. Biochem. Pharmacol, 177, 113954 (2020).

14. Tomás, J., Santos, C. R. A., Quintela, T. \& Gonçalves, I. "Tasting” the cerebrospinal fluid: Another function of the choroid plexus?, 320, 160-171 (2016).

15. Ren, X., Zhou, L., Terwilliger, R. \& Newton, S. S. Araujo, I. E. Sweet taste signaling functions as a hypothalamic glucose sensor. Frontiers in Integrative Neuroscience, 3, 12 (2009). de

16. Schiöth, H. B. \& Lagerström, M. C. Structural diversity of g proteincoupled receptors and significance for drug discovery. Nat. Rev. Drug Discovery, 7, 339-357 (2008).

17. Dagan-Wiener, A. et al. Bitterdb: Taste ligands and receptors database in 2019. Nucleic Acids Res, 47, D1179-D1185 (2019).

18. Magklara, A. et al. An epigenetic signature for monoallelic olfactory receptor expression., $\mathbf{1 4 5}, 555-$ 570 (2011).

19. Vakoc, C. R., Mandat, S. A., Olenchock, B. A. \& Blobel, G. A. Histone H3 lysine 9 methylation and HP1Y are associated with transcription elongation through mammalian chromatin. Mol. Cell, 19, 381-391 (2005).

20. Squazzo, S. L. et al. Suz12 binds to silenced regions of the genome in a cell-type-specific manner. Genome Res, 16, 890-900 (2006).

21. Wiencke, J. K., Zheng, S., Morrison, Z. \& Yeh, R. F. Differentially expressed genes are marked by histone 3 lysine 9 trimethylation in human cancer cells., 27, 2412-2421 (2008).

22. Dai, Z. et al. Insights into distinct regulatory modes of nucleosome positioning. BMC Genomics, 10, 602 (2009).

23. Berretta, J. \& Morillon, A. Pervasive transcription constitutes a new level of eukaryotic genome regulation. EMBO Reports, 10, 973-982 (2009).

24. Saint-André, V., Batsché, E., Rachez, C. \& Muchardt, C. Histone H3 lysine 9 trimethylation and HP1Y favor inclusion of alternative exons. Nature Structural and Molecular Biology, 18, 337-344 (2011).

25. Bieberstein, N. I. et al. TALE-directed local modulation of H3K9 methylation shapes exon recognition. Sci. Rep, 6, 29961 (2016).

26. Barrand, S., Andersen, I. S. \& Collas, P. Promoter-exon relationship of H3 lysine 9, 27, 36 and 79 methylation on pluripotency-associated genes. Biochemical and Biophysical Research Communications, 401, 611-617 (2010).

27. Spies, N., Nielsen, C. B., Padgett, R. A. \& Burge, C. B. Biased Chromatin Signatures around Polyadenylation Sites and Exons. Mol. Cell, 36, 245-254 (2009). 
28. Schwartz, S., Meshorer, E. \& Ast, G. Chromatin organization marks exon-intron structure. Nature Structural and Molecular Biology, 16, 990-995 (2009).

29. Andersson, R., Enroth, S., Rada-Iglesias, A., Wadelius, C. \& Komorowski, J. Nucleosomes are well positioned in exons and carry characteristic histone modifications. Genome Res, 19, 1732-1741 (2009).

30. Tilgner, H. et al. Nucleosome positioning as a determinant of exon recognition. Nature Structural and Molecular Biology, 16, 996-1001 (2009).

31. Nahkuri, S., Taft, R. J. \& Mattick, J. S. Nucleosomes are preferentially positioned at exons in somatic and sperm cells., 8, 3420-3424 (2009).

32. Mi, H. et al. Protocol Update for large-scale genome and gene function analysis with the PANTHER classification system (v.14.0). Nat. Protoc, 14, 703-721 (2019).

33. Thambirajah, A. A. et al. MeCP2 binds to nucleosome free (linker DNA) regions and to H3K9/H3K27 methylated nucleosomes in the brain. Nucleic Acids Res, 40, 2884-2897 (2012).

34. Dandrea, M., Donadelli, M., Costanzo, C., Scarpa, A. \& Palmieri, M. MeCP2/H3meK9 are involved in IL6 gene silencing in pancreatic adenocarcinoma cell lines. Nucleic Acids Res, 37, 6681-6690 (2009).

35. Lee, W., Kim, J., Yun, J. M., Ohn, T. \& Gong, Q. MeCP2 regulates gene expression through recognition of H3K27me3. Nat. Commun, 11, 3140 (2020).

36. Keller, A., Zhuang, H., Chi, Q., Vosshall, L. B. \& Matsunami, H. Genetic variation in a human odorant receptor alters odour perception., 449, 468-472 (2007).

37. Vest, R. S. \& Pike, C. J. Gender, sex steroid hormones, and Alzheimer's disease. Hormones and Behavior, 63, 301-307 (2013).

38. Gaudel, F., Guiraudie-Capraz, G. \& Féron, F. Limbic expression of mRNA coding for chemoreceptors in human brain-lessons from brain atlases. International Journal of Molecular Sciences22, (2021)

39. Freund, J. R. et al. Activation of airway epithelial bitter taste receptors by pseudomonas aeruginosa quinolones modulates calcium, cyclic-amp, and nitric oxide signaling. Journal of Biological Chemistry, 293, 9824-9840 (2018).

40. Zhou, L. et al. Sweet taste receptors mediated ROS-NLRP3 inflammasome signaling activation: Implications for diabetic nephropathy. Journal of Diabetes Research 2018, 7078214 (2018)

41. Zhao, W. et al. Decreased level of olfactory receptors in blood cells following traumatic brain injury and potential association with tauopathy. Journal of Alzheimer's Disease, 34, 417-429 (2013).

42. Breiling, A., Turner, B. M., Bianchi, M. E. \& Orlando, V. General transcription factors bind promoters repressed by Polycomb group proteins., 412, 651-655 (2001).

43. Dellino, G. I. et al. Polycomb silencing blocks transcription initiation. Mol. Cell, 13, 887-893 (2004).

44. Soufi, A., Donahue, G. \& Zaret, K. S. Facilitators and impediments of the pluripotency reprogramming factors' initial engagement with the genome., 151, 994-1004 (2012).

45. Lithner, C. U. et al. Disruption of neocortical histone $\mathrm{H} 3$ homeostasis by soluble Aß: Implications for Alzheimer's disease.Neurobiology of Aging34, (2013) 
46. Zheng, Y. et al. Inhibition of EHMT1/2 rescues synaptic and cognitive functions for Alzheimer's disease., 142, 787-807 (2019).

47. Lee, M. Y. et al. Epigenome signatures landscaped by histone H3K9me3 are associated with the synaptic dysfunction in Alzheimer's disease., 19, e13153 (2020).

48. Hernández-Ortega, K., Garcia-Esparcia, P., Gil, L., Lucas, J. J. \& Ferrer, I. Altered Machinery of Protein Synthesis in Alzheimer's: From the Nucleolus to the Ribosome.Brain Pathology26, (2016)

49. Frost, B., Hemberg, M., Lewis, J. \& Feany, M. B. Tau promotes neurodegeneration through global chromatin relaxation. Nature Neuroscience17, (2014)

50. Olender, T. et al. The human olfactory transcriptome. BMC Genomics, 17, 619 (2016).

51. 51. Livak, K. J. \& Schmittgen, T. D. Analysis of relative gene expression data using real-time quantitative PCR and the 2- $\triangle \Delta C T$ method. Methods 25, 402-408 (2001).

52. Donovan, L. L. \& Lichota, J. Native Chromatin Immunoprecipitation from Brain Tissue Using Magnetic Beads. Med. Chem. (Los. Angeles). 4, 738-741 (2014).

53. Jorge, I. et al. High-sensitivity analysis of specific peptides in complex samples by selected MS/MS ion monitoring and linear ion trap mass spectrometry: Application to biological studies. J. Mass Spectrom. 42, 1391-1403 (2007).

\section{Figures}

a

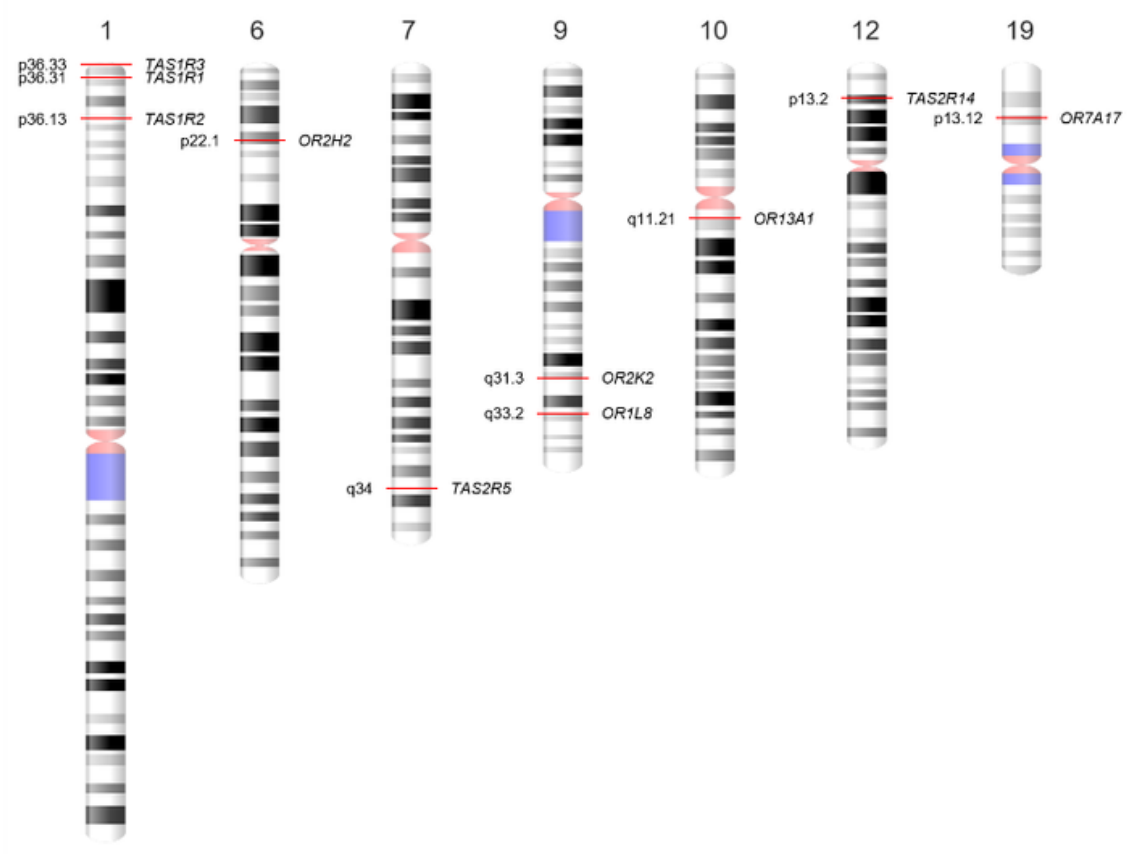

b

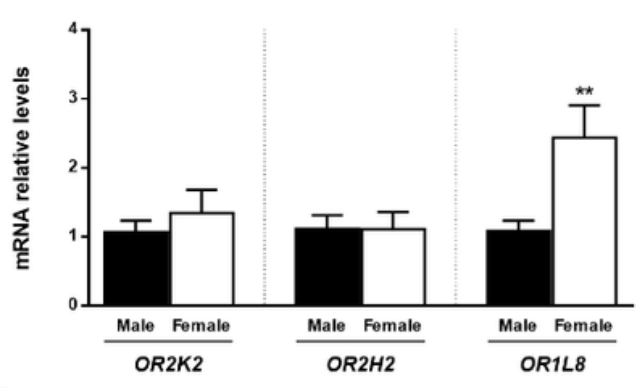

C

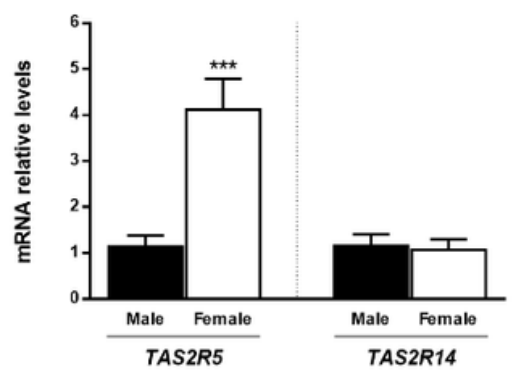


Ideogram of the distribution of the selected OR and TASR genes on the human genome (a) and ORs (b) and TAS2Rs (c) mRNA relative levels in OFC specimens from non-demented control samples. a. Red lines indicate the genomic location of each selected gene, the cytogenetic band of each locus is shown on the left, and its corresponding gene name (symbol) is indicated on the right. Chromosome number is presented on top. The cytogenetic representation of the selected chromosomes of the human genome (GRCh38.p12) was adapted from NCBI's Genome Decoration page (https://www.ncbi.nlm.nih.gov/genome/tools/gdp). b, c. ORs (b) and TAS2Rs (c) mRNA relative levels were normalized to GAPDH. Error bars represent standard error of the mean. Nonparametric MannWhitney U test (two-tailed) was used to compare the differences in the transcripts' relative levels between male $(n=13)$ and female $(n=12)$ groups. Statistical significance is expressed as ** $P<0.01$, and $\star \star \star P<$ 0.001 compared to male group.

a

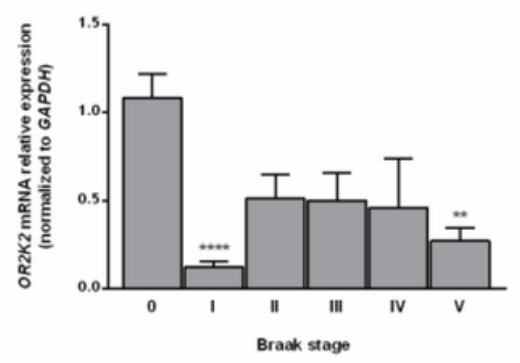

d

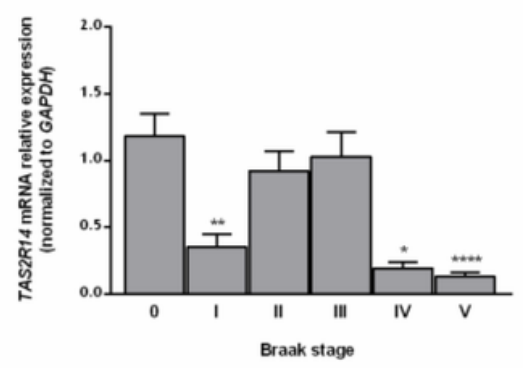

b

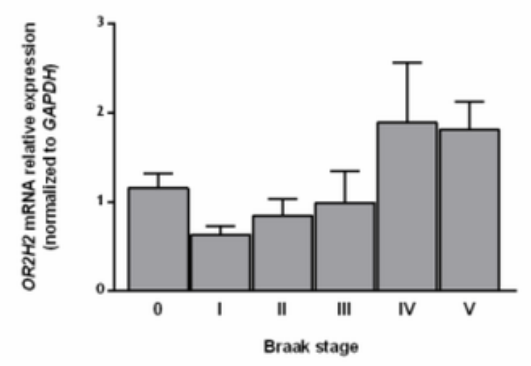

e

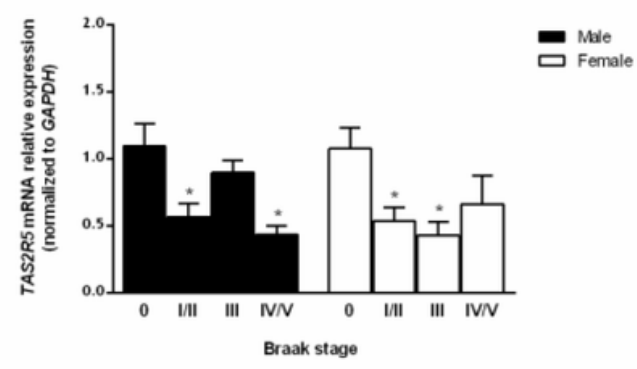

Figure 2

Olfactory and Taste Receptors mRNA relative expression on post-mortem OFC specimens from several Braak stages of sporadic AD compared to age-matched non-demented controls. a. OR2K2. b. OR2H2. c. OR1L8 separated by sex. d. TAS2R14. e. TAS2R5 separated by sex. mRNA relative levels were normalized to GAPDH. Error bars represent standard error of the mean. Statistical significance is expressed as * $P<$

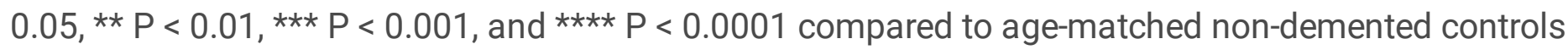
(Braak stage 0 ), Kruskal-Wallis $\mathrm{H}$ test followed by Dunn's multiple comparisons post-hoc test. Sporadic AD samples, $n=49$ and age-matched non-demented controls, $n=25$. 


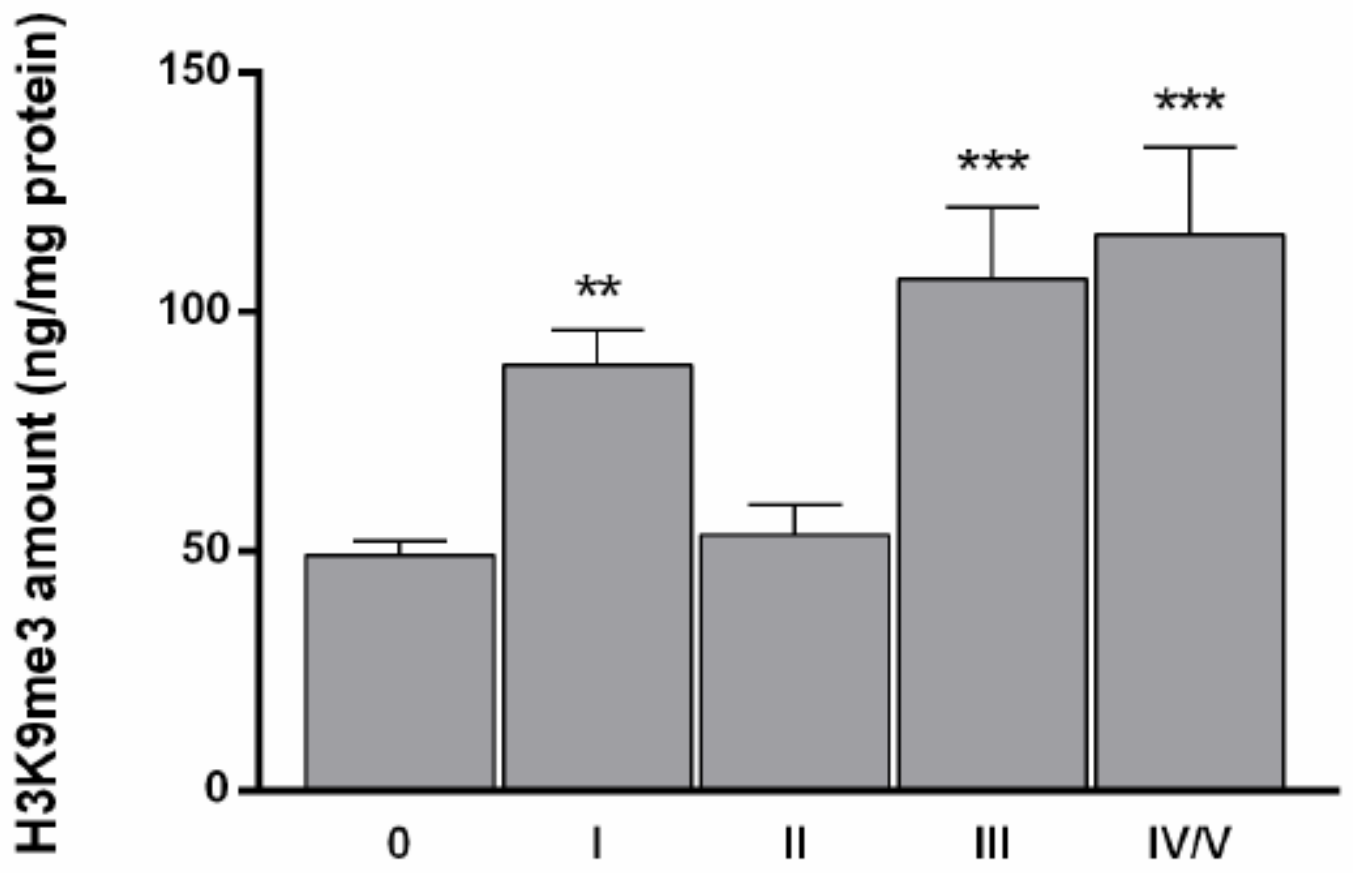

Braak stage

Figure 3

Global H3K9me3 amount (ng/mg protein) on post-mortem OFC histone extracts from sporadic AD. Error bars represent standard error of the mean. Statistical significance is expressed as $* * P<0.01$, and $* \star \star P<$ 0.001 compared to age-matched non-demented controls (Braak stage 0 ), Kruskal-Wallis $\mathrm{H}$ test followed by Dunn's multiple comparisons post-hoc test. Sporadic AD, $n=49$ and age-matched non-demented controls, $\mathrm{n}=24$. 
a

$\begin{array}{lllll}\text { OR2H2 } & \text { OR2K2 } & \text { OR1L8 } & \text { TAS2R14 } & \text { TAS2R5 }\end{array}$

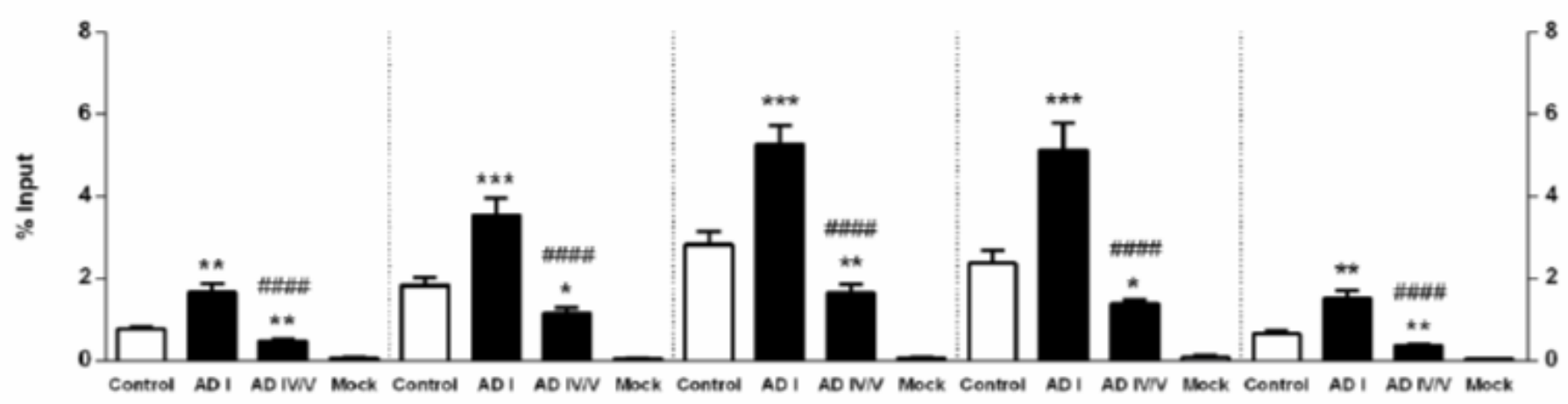

b
$\mathrm{OR} 2 \mathrm{H} 2$
OR2K2
OR1L8
TAS2R14
TAS2R5

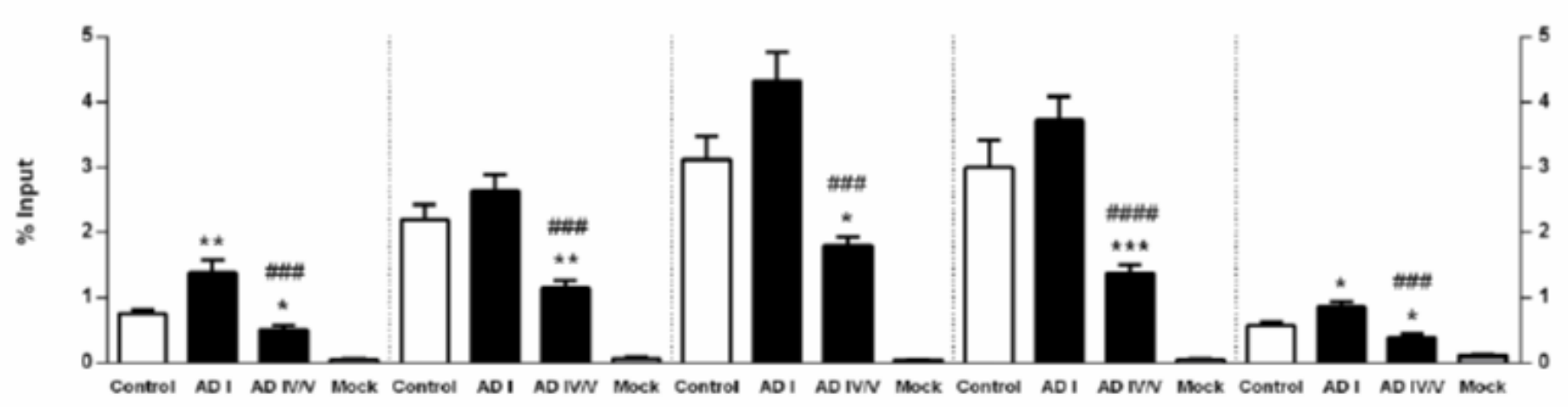

Figure 4

Native chromatin immunoprecipitation (N-ChIP) and quantitative real-time PCR analysis of H3K9me3 at each genomic location of interest. a. Proximal promoter of each receptor gene. b. Coding region of each receptor gene. Results are reported as the percentage of the input chromatin that is precipitated at each region of interest. Mock denotes negative control. Error bars represent standard error of the mean.

Statistical significance is expressed as $* P<0.05$, $* * P<0.01$, and $* \star * P<0.001$ compared to control, and as \#\#\# $\mathrm{P}<0.001$, and \#\#\#\# $\mathrm{P}<0.0001$ compared to Braak stage I group, Mann-Whitney $U$ tests (twotailed). Non-demented controls, $n=15 ;$ Braak I, $n=14$ and Braak IV/V, $n=10$. 
a

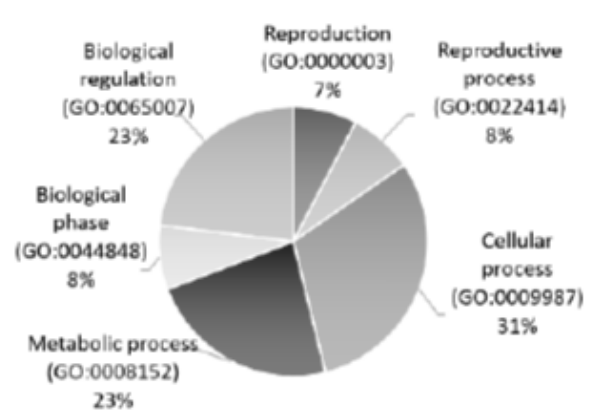

b

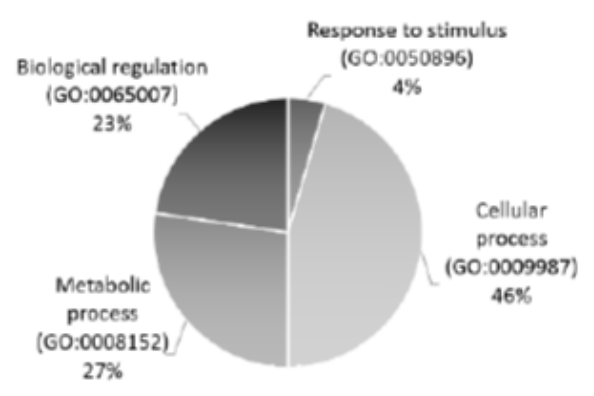

C

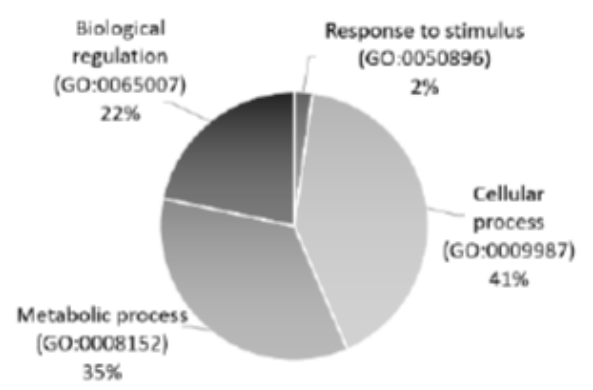

d

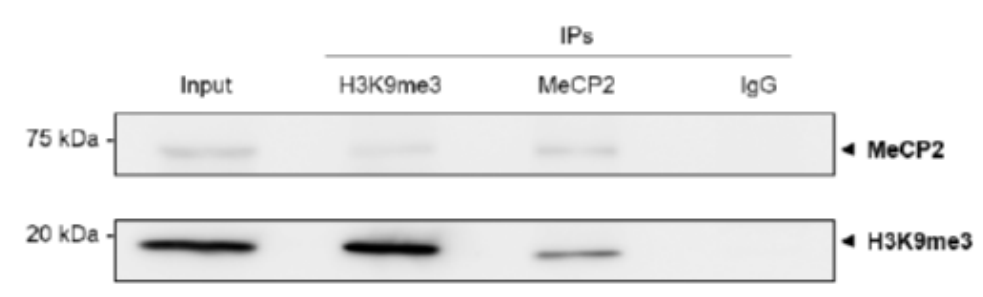

f

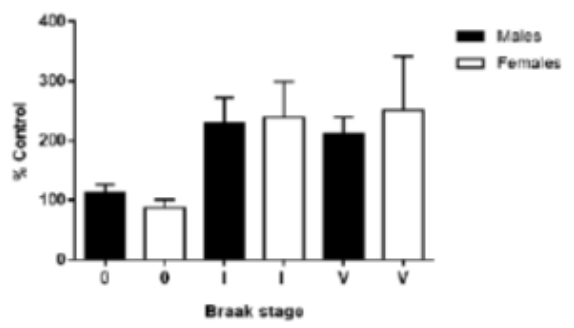

e

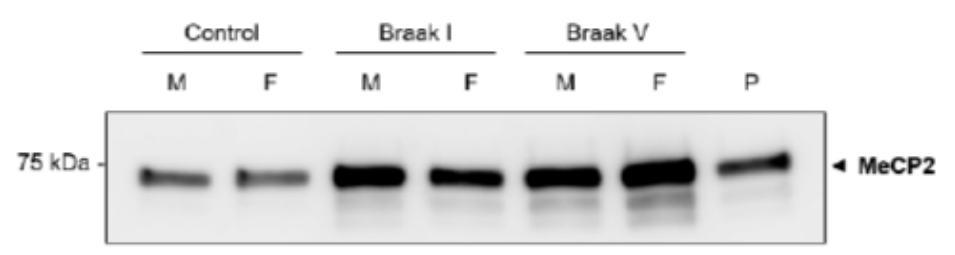

g

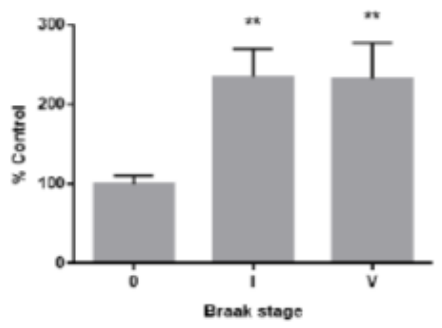

\section{Figure 5}

H3K9me3 interactome identification by mass spectrometry-based proteomics and coimmunoprecipitation validation. a, b, c. H3K9me3 immunoprecipitation was performed in pooled samples from Braak I and V stages, and proteins identified by mass spectrometry-based proteomics. Gene ontology (GO) annotation on biological process for proteins identified uniquely in Braak I (a), common to Braak I and V (b) and Braak V only (c). d. Validation of H3K9me3-MeCP2 interaction by reciprocal nuclear complex co-immunoprecipitations. Nuclear fractions from OFC specimens of 3 pooled Braak I (male samples) were used for $\mathrm{H} 3 \mathrm{~K} 9 \mathrm{me} 3, \mathrm{MeCP} 2$, and IgG negative control IP. The blots were cropped and fulllength blots are presented in Supplementary Figure 2. e. Representative immunoblot of MeCP2 protein expression in nuclear fractions from pooled OFC samples. The blots were cropped and full-length blots are presented in Supplementary Figure 2. A pool of samples $(P)$ was loaded to compare across different assays. M, Male; F, Female. f, g. Quantification of MeCP2 protein levels separated by sex (f) and combined (g). Stain-free total protein was used for total protein normalization. Statistical significance is expressed as ${ }^{\star *} \mathrm{P}<0.01$ compared to control (Braak stage 0 ), Kruskal-Wallis $\mathrm{H}$ test followed by Dunn's multiple comparisons post-hoc test. Non-demented controls, $n=18 ;$ Braak $I, n=15$ and Braak V, $n=9$.

\section{Supplementary Files}


This is a list of supplementary files associated with this preprint. Click to download.

- AlvesetalSciRepGraphicalabstract.png

- AlvesetalSciRepSupplementaryFigures.docx

- AlvesetalSciRepSupplementaryTables.pdf 